\title{
An optimisation strategy for industrial metal forming processes
}

\author{
Modelling, screening and solving of optimisation problems in metal forming
}

\author{
M. H. A. Bonte • A. H. van den Boogaard • J. Huétink
}

Received: 29 September 2006 / Accepted: 7 September 2007 / Published online: 18 March 2008

(C) The Author(s) 2008

\begin{abstract}
Product improvement and cost reduction have always been important goals in the metal forming industry. The rise of finite element (FEM) simulations for processes has contributed to these goals in a major way. More recently, coupling FEM simulations to mathematical optimisation techniques has shown the potential to make a further giant contribution to product improvement and cost reduction. Much research on the optimisation of metal forming processes has been published during the last couple of years. Although the results are impressive, the optimisation techniques are generally only applicable to specific optimisation problems for specific products and specific metal forming processes. As a consequence, applying optimisation techniques to other metal forming problems requires a lot of optimisation expertise, which forms a barrier for more general industrial application of these techniques. In this paper, we overcome this barrier by proposing a generally applicable optimisation strategy that makes use of FEM simulations of metal forming processes. It consists of a structured methodology for modelling optimisation problems related to metal forming. Subsequently, screening is applied to reduce the size of the optimisation problem by selecting only the most important design variables. Finally, the reduced optimisation problem is solved by an efficient optimisation algorithm. The strategy is generally applicable in a sense that it is not constrained to a certain type of metal forming problem, product or process. Also, any FEM code may be included in the strategy. Furthermore,
\end{abstract}

M. H. A. Bonte $(\varangle)$ · A. H. van den Boogaard · J. Huétink University of Twente, P.O. Box 217,

NL-7500 AE Enschede, The Netherlands

e-mail:m.h.a.bonte@utwente.nl the structured approach for modelling and solving optimisation problems should enable non-optimisation specialists to apply optimisation techniques to improve their products and processes. The optimisation strategy has been successfully applied to a hydroforming process, which demonstrates the potential of the optimisation of metal forming processes in general and more specific the proposed optimisation strategy.

Keywords Metal forming • Finite element method • Optimisation

\section{Introduction}

During the past few decades, finite element (FEM) simulations of metal forming processes have become important tools for designing feasible production processes. In more recent years, coupling FEM simulations to mathematical optimisation techniques evolved to address two industrial needs: (1) designing optimal metal forming processes instead of only feasible ones (better products, lower costs) and (2) solving problems in manufacturing.

The basic concept of mathematical optimisation is presented in Fig. 1. Basically, it consists of two major phases: the modelling and the solving of the optimisation problem. The modelling phase consists of:

1. Selecting a number of design variables the user is allowed to adapt

2. Choosing an objective function, i.e. the optimisation aim

3. Taking into account possible constraints 


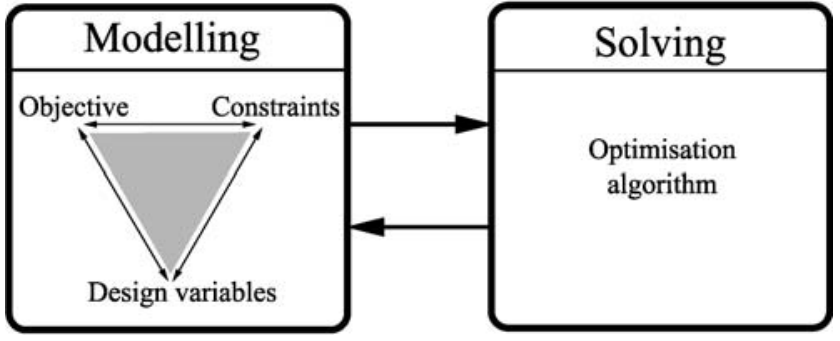

Fig. 1 The basic concept of mathematical optimisation: modelling and solving

The solving phase comprises applying a suitable optimisation algorithm to the modelled optimisation problem.

The arrows between the modelling and the solving parts in Fig. 1 denote that both phases cannot be seen separately from each other. One should select the right optimisation algorithm for a certain modelled optimisation problem and one should model the optimisation problem cleverly to adjust it to the optimisation algorithm one is planning to apply. If the optimisation model does not match the algorithm, it is likely that the optimisation problem is not solved efficiently or cannot be solved at all (Papalambros and Wilde 2000).

Academic research on the field of optimisation of metal forming using time-consuming FEM simulations has been going on now for several years, yielding a large number of scientific publications. Ben Ayed et al. (2004a, b, 2005), Breitkopf et al. (2005), Cao et al. (2001), Debray et al. (2004), Jansson (2002), Jansson et al. 2005, Kim et al. (2001a), Kleinermann (2001), Kleinermann and Ponthot (2003), Lenoir and Boudeau (2003), Liew et al. (2004), Naceur et al. (2001, 2003, 2004a, b, c), Ponthot and Kleinermann (2005), Schenk and Hillmann (2004), Sheng et al. (2004), Shi et al. (2004), Lin et al. (2002) and Strano and Carrino (2004) apply optimisation techniques to deep drawing; Abedrabbo et al. (2004), Aydemir et al. (2005), Di Lorenzo et al. (2004), Endelt (2003), Endelt and Nielsen (2001), Fann and Hsiao (2003), Jirathearanat and Altan (2004), Johnson et al. (2004), Labergère and Gelin (2004a, b), Ray and Mac Donald (2004), Sillekens and Werkhoven (2001), Weyler et al. (2004) and Yang et al. (2001) to hydroforming; Carrino et al. (2003a, b) and Kim et al. (2001b) to superplastic forming; Byon and Hwang (2001) and Lin et al. (2003) to extrusion; and António et al. (2004), Castro et al. (2004), Do et al. (2004), Duan and Sheppard (2002), Fourment et al. (2005a, b), Poursina et al. (2003), Repalle and Grandhi (2004), Sousa et al. (2003), Thiyagarajan and Grandhi (2004) and Zhao et al. (2004) to forging. Other processes are subject to optimisation in Breitkopf et al.
(2004), Labergère et al. (2004), Ohata et al. (2003), Palaniswamy et al. (2004) and Sahai et al. (2004).

In general, one can conclude that most research until now has been focussing on the solving part of optimisation, i.e. the selection and application of a suitable optimisation algorithm. Modelling is mostly done in an arbitrary way, addressing the specific problem of the considered metal forming process only. Furthermore, the selection of the optimisation algorithm is also mainly related to that specific problem.

Most optimisation results are very impressive, which demonstrates the large potential of optimisation techniques to optimise metal forming processes. However, modelling an optimisation problem well, selecting a suitable optimisation algorithm, and proper application of this algorithm to a specific metal forming problem requires a lot of expertise on mathematical optimisation. Unfortunately, most professionals in metal forming do not have this expertise, which is a barrier for exploiting the full potential of optimising metal forming processes.

To overcome this barrier, there is a need for a generally applicable optimisation strategy for metal forming processes: a structured method that assists metal forming professionals in modelling and solving a variety of metal forming problems, regardless of the type of problem, product or process they are facing. Such an optimisation strategy is presented in this paper.

In the next section, certain aspects of mathematical optimisation using time-consuming FEM simulations will be reviewed first. In Section 3, an optimisation strategy for metal forming processes is proposed. This strategy is applied to a hydroforming example in Section 4. Section 5 draws some conclusions.

\section{Mathematical optimisation of metal forming using FEM}

The basic concept of mathematical optimisation has been presented in Fig. 1. This section covers some aspects of the application of mathematical optimisation to metal forming processes using time-consuming FEM simulations. Both the modelling and solving parts are addressed.

\subsection{Modelling}

Two aspects of modelling are treated: the effect of arbitrary modelling on the optimised results and the relation between optimisation modelling and FEM simulations. 
The effect of arbitrary modelling As mentioned before, the optimisation modelling comprises defining the objective function, constraints and design variables. These three items are closely related to each other as depicted in Fig. 1. Both the objective function and the constraints should be quantified by the design variables. The objective function and constraints are also related to each other in the sense that they are often exchangeable. Consider for example that we would like to make a metal formed product and two relevant properties are the product quality and the costs. Then two approaches can be followed: either the quality is maximised while putting a certain limit on the allowed production costs, or the costs could be minimised while ensuring a certain minimum level of the product quality. In the former case, the quality is clearly the optimisation objective and the costs are constraints, whereas it is just the other way around in the latter case. Which property to select as an objective and which as a constraint is a very fundamental question because it strongly influences the final outcome of optimisation. Moreover, coincidentally missing an essential property to take into account may have devastating consequences for the optimised results: minimising the costs while leaving out the quality in the above example will yield a very cheap but very poor product regarding quality.

The relation between optimisation and FEM Figure 2 schematically presents the relation between optimisation and FEM. Running a FEM simulation can be seen as an input-throughput-response model. Certain quantities are known beforehand: there is no necessity to run a FEM calculation for evaluating them. The design variables are clear examples of these quantities and there can also be constraints that explicitly depend on the design variables. These constraints are called explicit constraints. In case of metal forming, explicit constraints are related to the undeformed product, e.g. constraints on the initial shape of a blank.

Quantities that depend on the response require a FEM simulation for evaluating them: they implicitly depend on the design variables. The objective function is generally such an implicit quantity and it is also possible to have implicit constraints. For metal forming, implicit constraints are related to the deformed product, e.g. thinning is not allowed to exceed a specified limit.

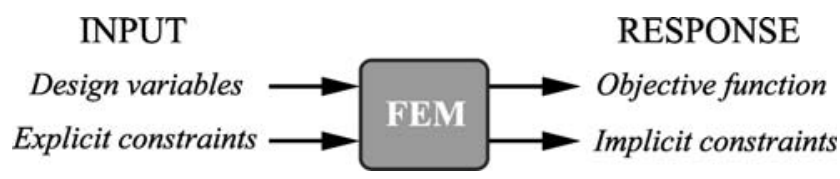

Fig. 2 FEM as an input-throughput-response model

\subsection{Solving}

Next to the modelling phase, mathematical optimisation's second phase is solving the optimisation problem. This comprises applying an optimisation algorithm to the modelled optimisation problem.

Scientific publications mainly focus on this solving part of mathematical optimisation. In the remainder of this section, we will review four major groups of algorithms that can be applied for optimisation using FEM simulations:

- Iterative algorithms

- Evolutionary and genetic algorithms

- Approximate optimisation algorithms

- Adaptive optimisation algorithms

We will also indicate the extensive application of these groups of algorithms to optimise metal forming processes.

Iterative algorithms One way of optimising metal forming processes is using classical iterative optimisation algorithms (conjugate gradient, BroydenFletcher-Goldfarb-Shanno algorithm, etc.). Using these iterative algorithms, there is a direct coupling between the FEM software and the optimisation algorithm as depicted in Fig. 3a: each function evaluation of the algorithm means running a FEM calculation. In case of metal forming, these FEM calculations can be extremely time consuming and need to be sequentially evaluated. Furthermore, many classical algorithms require sensitivities, of which the efficient calculation is not straightforward for FEM simulations. A third difficulty concerning iterative algorithms is the risk to be trapped in local optima. Advantages of this group of algorithms comprise the fact that they are well-known and widely spread. Additionally,
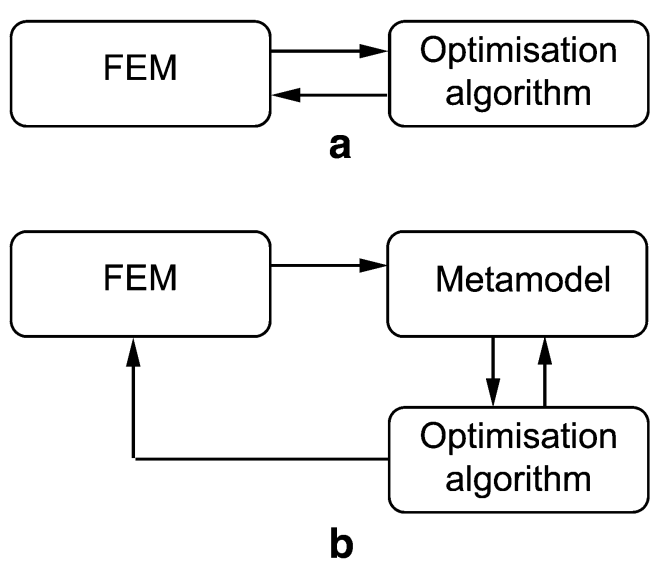

Fig. 3 a Direct optimisation; b approximate optimisation 
convergence to a local optimum generally requires relatively few iterations and is, hence, fairly efficient. Due especially to the former advantage, iterative algorithms are quite often applied to optimise metal forming processes, see, e.g. Byon and Hwang (2001), Cao et al. (2001), Debray et al. (2004), Duan and Sheppard (2002), Endelt and Nielsen (2001); Endelt (2003), Fann and Hsiao (2003), Jirathearanat and Altan (2004), Kim et al. (2001a, b), Kleinermann (2001), Kleinermann and Ponthot (2003), Lin et al. (2002, 2003), Naceur et al. (2001, 2004c), Ponthot and Kleinermann (2005), Shi et al. (2004), Sillekens and Werkhoven (2001), Yang et al. (2001), Zhao et al. (2004).

Evolutionary and genetic algorithms A second group of algorithms for which there is a direct coupling between the algorithm and the FEM software (Fig. 3a) are genetic and evolutionary optimisation algorithms. Genetic and evolutionary algorithms look promising because of their tendency to find the global optimum and the possibility for parallel computing. Furthermore, it is not difficult to calculate sensitivities with them. However, the rather large number of function evaluations that is expected to be necessary using these algorithms is regarded as a serious drawback (Emmerich et al. 2002). Several authors have applied genetic and evolutionary algorithms to optimise metal forming processes, see Abedrabbo et al. (2004), António et al. (2004), Castro et al. (2004), Do et al. (2004), Fourment et al. (2005a, b), Poursina et al. (2003), Schenk and Hillmann (2004), Sousa et al. (2003), and Weyler et al. (2004).

Approximate algorithms A third way of optimisation in combination with time-consuming function evaluations is using approximate optimisation algorithms, of which response surface methodology (RSM) is a wellknown representative. RSM is based on fitting a loworder polynomial metamodel through response points, which are obtained by running FEM calculations for carefully chosen design variable settings and finally optimising this metamodel (Myers and Montgomery 2002). Hence, for approximate optimisation, the direct coupling between the optimisation algorithm and the FEM calculations is removed and a metamodel is placed in between as a buffer. This is schematically presented in Fig. 3b. Metamodels are sometimes also referred to as response surface models or surrogate models. Next to RSM, other metamodelling techniques are Kriging [or design and analysis of computer experiments (DACE)] and neural networks. Allowing for parallel computing and lacking the necessity for sensitivities, approximate optimisation is appealing to many authors in the field of metal forming, see Ben Ayed et al. (2004a, b, 2005), Breitkopf et al. (2004, 2005), Jansson (2002), Jansson et al. (2005), Koc et al. (2000), Lenoir and Boudeau (2003), Liew et al. (2004), Naceur et al. (2003, 2004a, b), Ohata et al. (2003), Repalle and Grandhi (2004), Revuelta and Larkiola (2004), Sahai et al. (2004), and Thiyagarajan and Grandhi (2004). Disadvantages include an approximate optimum as a result rather than the real global optimum and the curse of dimensionality: these algorithms tend to become very time consuming if many design variables are present.

Adaptive algorithms A fourth group is formed by socalled adaptive algorithms. Adaptive algorithms are not coupled to FEM in the same way as the other three groups of algorithms. Adaptive algorithms are incorporated within the FEM code and generally optimise the time-dependent load paths of the metal forming process during each increment of the FEM calculation. An advantage is that the optimum is obtained in only one FEM simulation. However, access to the source code of the FEM software is necessary and only timedependent design variables can be taken into account. These disadvantages seriously limit the general applicability of these kinds of algorithms. Literature describes several applications of these algorithms to metal forming (Aydemir et al. 2005; Carrino et al. 2003a, b; Di Lorenzo et al. 2004; Johnson et al. 2004; Labergère et al. 2004; Labergère and Gelin 2004a, b; Ray and Mac Donald 2004; Sheng et al. 2004; Sillekens and Werkhoven 2001; Strano and Carrino 2004) especially to optimise the internal pressure and axial feeding load paths in hydroforming.

All groups of optimisation algorithms introduced in the previous section have been applied to optimisation problems in metal forming. In general, one can conclude from literature that specific problems for specific metal forming processes are - sometimes quite arbitrarily - modelled and subsequently solved using an algorithm suitable for that specific application. In our opinion, a generally applicable optimisation strategy for modelling and solving optimisation problems in metal forming problems is lacking. As a consequence, we developed such an optimisation strategy which can be applied to model and solve all kinds of optimisation problems for all kinds of metal forming processes using any simulation code. 


\section{Optimisation strategy for industrial metal forming processes}

In this section, we will propose an optimisation strategy for industrial metal forming processes. In Section 3.1, the requirements for the optimisation strategy are shortly introduced. Subsequently, the optimisation strategy is presented in Sections 3.2 to 3.4.

\subsection{Requirements}

Our aim has been to develop an optimisation strategy for metal forming processes using FEM models of these processes, but which processes? For which (type of) product? And which FEM codes should be included? We have tried to develop a strategy which is as generally applicable as possible. In this context, general applicability has four dimensions, as schematically presented in Fig. 4:

- Processes: the strategy should be able to model and solve all kinds of optimisation problems for all kinds of different metal forming processes.

- Products: the same holds for different products. The products mentioned in Fig. 4 are all deepdrawn products, but quite different demands are set for them.

- Users: although both can be related to metal forming, a product designer is confronted with totally different challenges from those of a manufacturing engineer.

- FEM codes: many FEM codes are available. The choice for specific simulation software depends heavily on the process, the product and the preference of the user.

Whereas the general applicability is the first requirement, a second requirement is the application of mathematical optimisation techniques: the modelling needs to result in a specific, mathematically formulated opti-

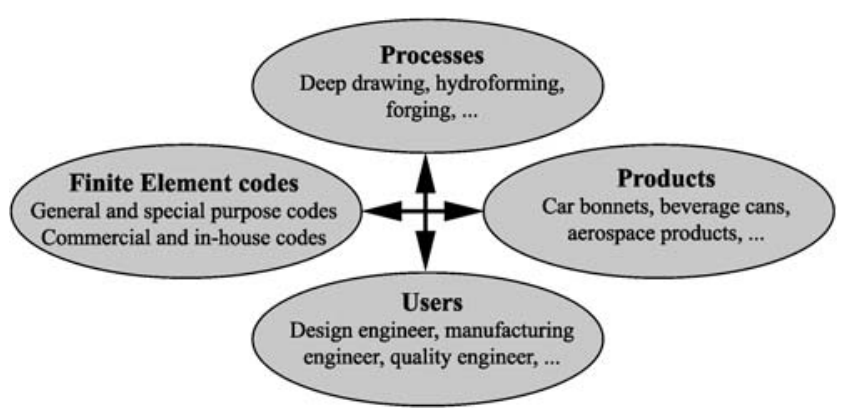

Fig. 4 General applicability of the optimisation strategy misation problem to subsequently solve it by a mathematical optimisation algorithm. Both requirements are quite contradictory: the optimisation strategy needs to be generally applicable, yet solving it mathematically requires a detailed and specific optimisation model.

We propose a three-stage optimisation strategy that lives up to both of the above requirements:

1. Modelling the optimisation problem

2. Screening to determine the most important design variables

3. Solving the optimisation problem

The different stages are presented in Sections 3.2 to 3.4 .

\subsection{Modelling}

The first stage is to model the optimisation problem. It is quite a challenge to design a structured methodology that is, on the one hand, generally applicable to any kind of metal forming problem but, on the other hand, yields a specific mathematical formulation of the optimisation problem.

We adopted the following approach for tackling this problem:

1. Brainstorming for industrially relevant objectives, constraints and design variables.

2. Structuring these quantities by means of the generally applicable product development cycle.

3. Applying this product development cycle to metal products and their forming processes.

4. Defining a seven-step methodology for modelling optimisation problems. This methodology is generally applicable to any metal forming problem for any process, product, FEM code and user. However, after having followed the seven steps, it results in a specific mathematical optimisation model.

Brainstorming Several brainstorm sessions have been organised at several large metal forming companies. Different users joined the sessions and different products and processes have been considered. The brainstorming sessions resulted in a large number of industrially relevant objectives, constraints and design variables.

Product development cycle At the basis of the structured methodology for modelling optimisation problems for industrial metal forming processes is the product development cycle, which is a part of the product life cycle. A schematic of the product life cycle 


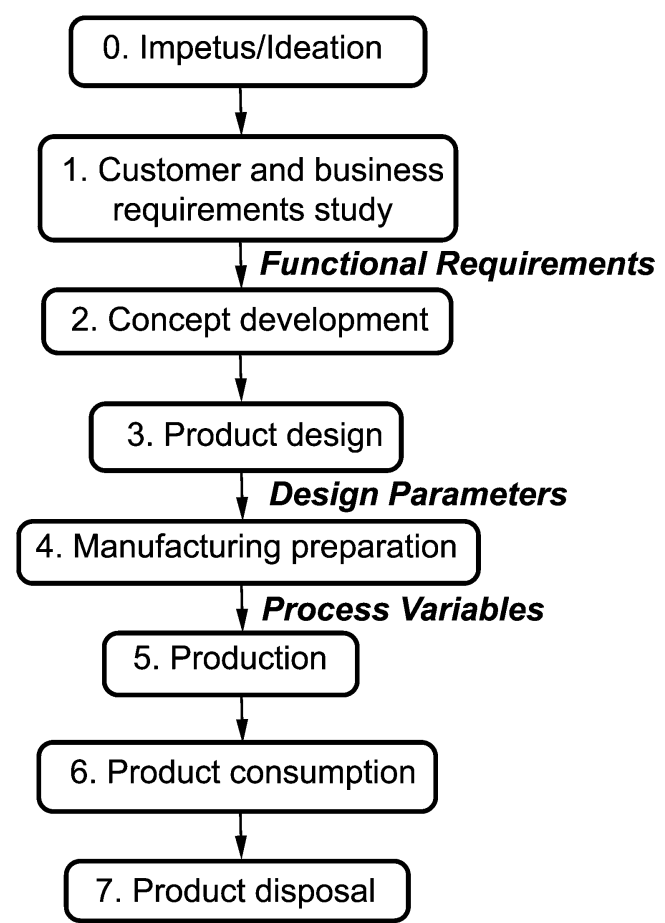

Fig. 5 The product life cycle

is presented in Fig. 5 (Yang and El-Haik 2003). The product development cycle is the stages 0 through 5, i.e. the product life cycle of Fig. 5 minus the stages 6 (product consumption) and 7 (product disposal).

Three groups of quantities are indicated in Fig. 5:

- Functional requirements (FRs): these are product properties that are critical to customer satisfaction and product functionality.

- Design parameters (DPs): these define the product design.

- Process variables (PVs): these are process settings necessary to manufacture the product.

The product life cycle and product development cycle are both very generally applicable. The next step is to make this generally applicable concept somewhat more specific by applying it to metal forming. This has been done by confronting the product development cycle to the metal forming quantities resulting from the brainstorm sessions.

Product development cycle applied to metal forming The industrially relevant metal forming quantities which are possible objective functions, constraints and design variables for optimisation - have been categorised in FRs, DPs and PVs. Two additional categories are costs and defects. They have been added to the product development cycle as presented in Fig. 6.

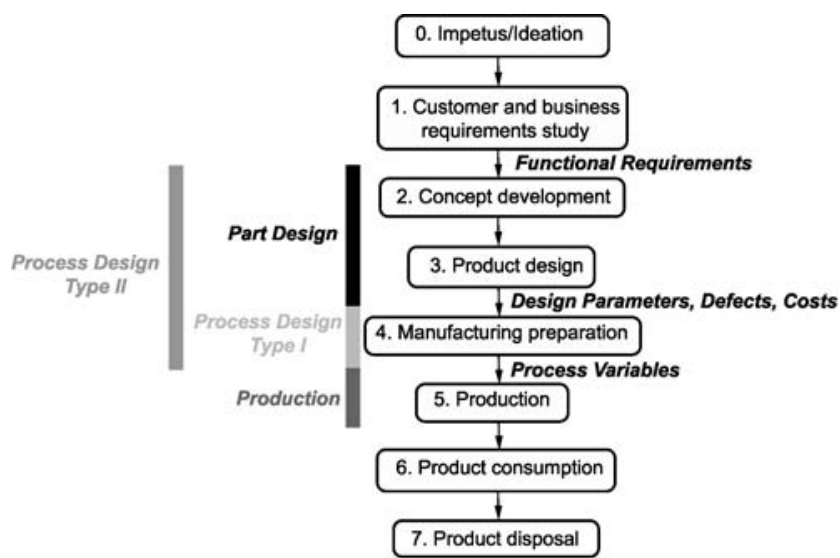

Fig. 6 The product life cycle applied to metal forming

Subsequently, top-down structures have been defined for each of the five categories. The top-down structures for the DPs and PVs are presented in Figs. 7 and 8, respectively. Typical examples of FRs for metal forming are crashworthiness properties, fatigue properties, stiffness, strength, etc. Examples of the category costs are material and process costs. Metal forming defects such as necking and wrinkling in sheet metal forming are comprised in the category defects.

7 step methodology The product development cycle applied to metal forming is the basis for a seven-step methodology for the modelling of optimisation problems in metal forming:

1. Determine the appropriate optimisation situation.

2. Select only the necessary responses.

3. Select one response as objective function, the others as implicit constraints.

4. Quantify the objective function and implicit constraints.

5. Select possible design variables.

6. Define the ranges on the design variables.

7. Identify explicit constraints.

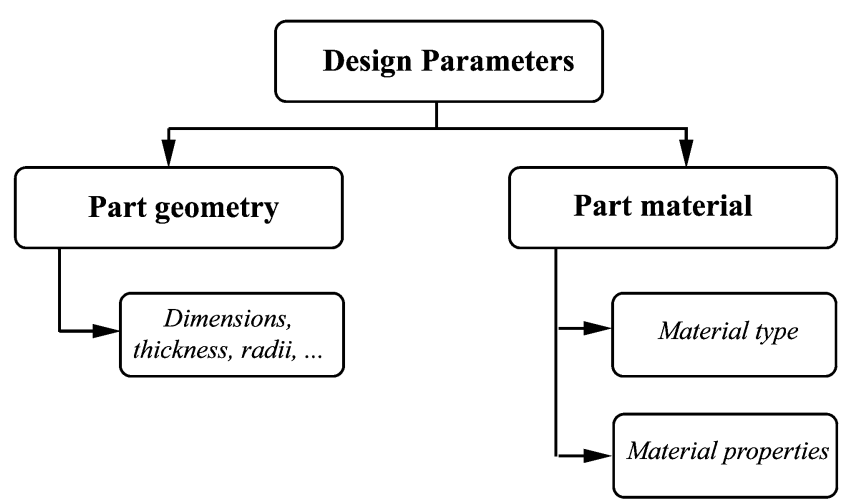

Fig. 7 Design parameters of metal formed parts 


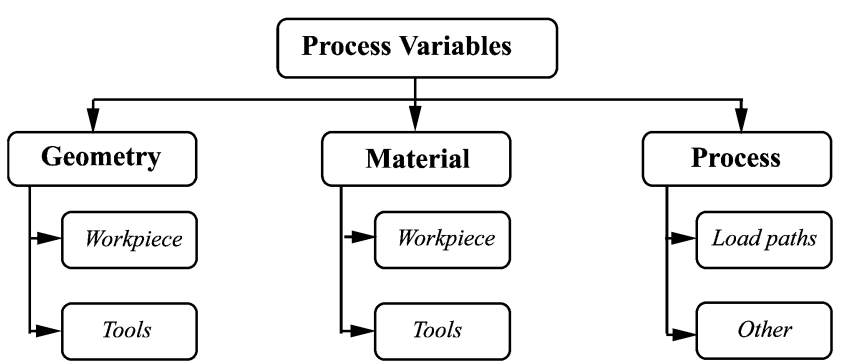

Fig. 8 Process variables for metal forming processes

The first step is to determine the appropriate optimisation situation. Four situations in which the optimisation of metal products and their manufacturing processes can play a role are distinguished:

- Part design, where it is aimed to optimise the metal formed product's FRs by determining the DPs.

- Process design type 1 , where it is aimed to exactly obtain the DPs set by the part designer by determining the PVs. Alternatively, one can aim to manufacture a defect-free product or minimise production costs by determining the PVs.

- Process design type 2, where it is aimed to optimise the metal formed product's FRs by determining the PVs. DPs, defects and costs can still play a role in parallel to the FRs.

- Production, where optimisation techniques can be used to solve manufacturing problems.

These situations and their relations to the product development cycle are presented in the left part of Fig. 6. To demonstrate how optimisation can be applied to one of these four situations, compare the inputresponse model for optimisation in Fig. 2 (repeated in Fig. 9) to that for a process design type 1 problem in Fig. 10. Note the resemblance: one can immediately observe that, for a process design type 1 problem, the objective function and implicit constraints are the DPs, defects and costs, whereas the design variables are related to the PVs.

In step 2 of the seven-step methodology, the topdown structures such as the ones in Figs. 7 and 8 can be used to select the necessary responses for the specific problem. These responses are output quantities of the FE simulation.

INPUT

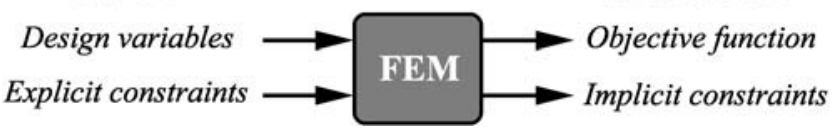

Fig. 9 Input-response model for FEM in relation to optimisation

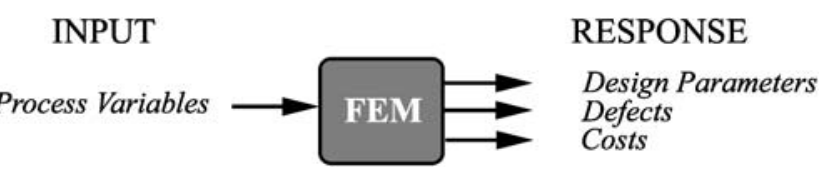

Fig. 10 Input-response model for FEM during the process design type 1 situation

According to step 3, one of the defined response quantities is selected as objective function, the others as implicit constraints. We prefer selecting one objective and defining other responses as constraints rather than multi-objective approaches such as a weighted sum of the responses. The latter approach compares, in our opinion, difficult to compare quantities and is somewhat arbitrary in the selection of weight factors.

Now, it is clear which FEM responses are formulated as objectives and which as constraints. However, the exact mathematical formulation of the responses is still not clear. This is done in step 4 of the seven-step methodology. For response quantification, Table 1 is proposed. It assists in selecting the final mathematical formulation of the objective function and implicit constraints. Objective functions are further subdivided in objectives that aim to reach a target and those that do not. Upper- and lower-limit implicit constraints are distinguished. Because the response quantity $X$ is a FEM output, it can be a nodal or element value or not. Examples of nodal/element values are strains, stresses, thickness, etc. Quantities such as forming energy are not nodal/element related: one number results from one FEM calculation. Furthermore, Table 1 subdivides the element/nodal value related responses further into critical and non-critical values. Critical values are the values for which none of the nodal/element values are allowed to exceed a specified level. If it is acceptable that some of the element/nodal values exceed this specified level, but important that the average response value performs well, the response is non-critical. Constraints are, by definition, critical values as one can see in Table 1.

Steps 5, 6 and 7 of the modelling methodology concern the FEM inputs, the design variables in case of optimisation. Step 5 comprises the design variable selection. The optimisation situation selected in step 1 determines the groups of design variables to be taken into account. For example, Fig. 10 shows that the PVs are the group of design variables for a process design type 1 situation. The top-down structure in Fig. 8 can subsequently be used to select the design variables for the specific optimisation problem.

Step 6 comprises the selection of the ranges (upper and lower bounds) on all design variables. This is 
Table 1 Response quantification

\begin{tabular}{llll}
\hline Type of response & No nodal/element value & Nodal/element value, critical & Nodal/element value, non-critical \\
\hline Objective function, no target & $\min X$ & $\min \max _{N} X$ & $\min \frac{\sum_{N}(X)}{N}$ \\
Objective function, target $=X_{0}$ & $\min \left|X-X_{0}\right|$ & $\min \max _{N}\left|X-X_{0}\right|$ & $\min \frac{\left\|X-X_{0}\right\|_{2}}{N}$ \\
Implicit constraint, USL & $X-U S L \leq 0$ & $\max _{N}(X-U S L) \leq 0$ & \\
Implicit constraint, LSL & $L S L-X \leq 0$ & $\max _{N}(L S L-X) \leq 0$ & \\
\hline
\end{tabular}

something that needs to be done based on the experience and insight of the user.

Step 7 concludes the input modelling by identifying explicit constraints. Explicit constraints describe impossible combinations of the design variables, e.g. it is not possible to run a FEM simulation for those settings, or the combination is beforehand an infeasible solution of the problem.

Without going into further detail, we conclude by reemphasising that this seven-step methodology is generally applicable to any metal forming problem and yields a specific mathematical optimisation model, which can subsequently be solved using a suitable optimisation algorithm. The seven-step methodology is demonstrated in the next section when it is applied to a simple hydroforming example.

\subsection{Screening}

After modelling, many design variables may be present, which makes the problem time-consuming to solve. It is worthwhile to invest some time in reducing the number of design variables before applying the optimisation algorithm. This is done in the screening stage.

We propose to screen the importance of the design variables by applying factorial DOE strategies (Myers and Montgomery 2002). Figure 11a shows a well-known full factorial design for two levels and three factors (design variables), a so-called $2^{3}$ full factorial design. It allows for estimation of the linear and interaction effects of the design variables and requires $2^{3}=8$ FEM calculations.

If one likes to estimate quadratic effects, a DOE of at least three levels is required. Figure $11 \mathrm{~b}$ shows a $3^{2}$ full factorial design for two design variables on three levels each. $3^{2}=9$ FEM calculations are required to estimate the linear, interaction and quadratic effects of the design variables. For more design variables, the number of FEM calculations explodes exponentially, which makes the application of full factorial designs prohibitively time-consuming.

However, if one is only interested in linear effects, the number of necessary FEM calculations can be sig- nificantly reduced by applying fractional factorial designs. Figure $11 \mathrm{c}$ shows a $2_{\mathrm{III}}^{3-1}$ fractional factorial DOE for three design variables. Resolution III denotes that it is possible to independently estimate the linear effects. Interaction effects cannot be independently estimated. The loss of information with respect to applying the $2^{3}$ full factorial design returns a decrease in the timeconsuming FEM simulations that need to be run: the $2_{\mathrm{III}}^{3-1}$ fractional factorial design requires only four FEM calculations in contradiction to the eight calculations for the full factorial design. For more design variables, the difference becomes significantly larger in favour of the resolution III fractional factorial designs.

For screening purposes, we are willing to consider linear effects only. Of course, neglecting interaction and other non-linear effects is a crude assumption, but the increase in efficiency is - at least during the screening stage - more important than the accuracy. Moreover, the amount of the linear effects should give an indication of the importance of the different design variables and can thus be used to omit the less important design variables.

After having applied the resolution III fractional factorial design and having run the corresponding FEM simulations, the linear effects can be estimated by applying statistical techniques such as analysis of variance (ANOVA), see e.g. Myers and Montgomery (2002). The amount and direction of the effect of each variable on each response can be nicely displayed in Pareto and effect plots (Yang and El-Haik 2003).

Using these techniques, the variables with the largest effects may be kept in the optimisation model, whereas the variables having less effect may be omitted. In

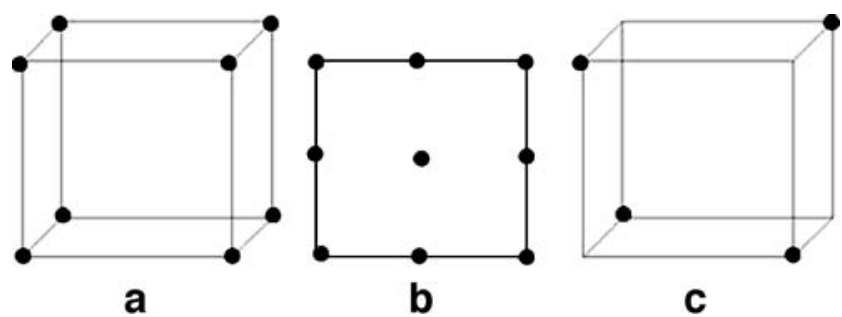

Fig. 11 a $2^{3}$ full factorial design; b $3^{2}$ full factorial design; c $2_{\text {III }}^{3-1}$ fractional factorial design 
such a way, the amount of design variables may be significantly decreased while maintaining control over objective function and constraints during optimisation. Screening and the use of Pareto and effect plots is further demonstrated in Section 4.

\subsection{Solving}

The final stage of the optimisation strategy is to solve the optimisation problem by a suitable algorithm.

Based on the literature study presented in Section 2.2 , we propose to use an approximate optimisation algorithm: it is efficient because several calculations can be run at the same time on parallel processors and it converges to the global optimum, which mostly results in better results than local algorithms. The disadvantage that this type of algorithm is not efficient in case many design variables are present is overcome by the screening stage of the optimisation strategy. Additionally, the algorithm is applicable to all kinds of metal forming processes, products and problems because the FEM simulations are included as a black-box.

An overview of the algorithm is presented in Fig. 12. Here, we will shortly summarise the different steps of the algorithm. For more details, refer to publications on the sequential approximate optimisation (SAO) algorithm (Bonte et al. 2005a, b, 2007; Bonte 2007).

DOE After having modelled - and perhaps screenedthe optimisation problem, the first step of the algorithm is to apply another DOE strategy. This DOE strategy indicates for which design variable settings

Fig. 12 Sequential approximate optimisation (Bonte et al. 2005a, b, 2007; Bonte 2007)

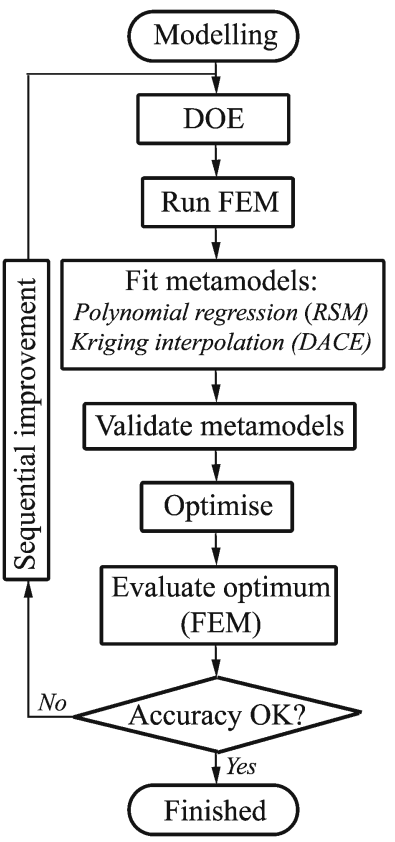

to run the first FEM simulations for optimisation. A spacefilling Latin hypercubes design (LHD) is a good and popular DOE strategy for constructing metamodels from deterministic computer experiments such as FEM calculations (McKay et al. 1979; Santner et al. 2003). The developed DOE takes into account explicit constraints and uses a so-called maximin criterion for spacefillingness (Bonte 2007).

Running the FEM simulations For the design variable settings determined by the LHD, FEM simulations are performed on parallel processors. Although FEM simulations for metal forming processes can be very timeconsuming, running the FEM simulations in parallel is quite time-efficient. Any FEM code for any product and any process may be applied, which guarantees the general applicability of the SAO algorithm.

Fitting the metamodels The FEM simulations result in a number of measurement points for each one of the modelled responses (objective function and implicit constraints). For each response, several metamodels are constructed using response surface methodology (RSM) (Myers and Montgomery 2002) and Kriging (Sacks et al. 1989a, b, Santner et al. 2003) metamodelling techniques. Both RSM and Kriging are statistical techniques: the metamodels of the responses can be interpreted as means. Additionally, it is also possible to determine a standard deviation at any design variable setting. To explain this, Fig. 13 presents a Kriging metamodel fitted through three response points $\mathbf{y}^{(i)}$ that resulted from running three FEM calculations for the design variable settings $\mathbf{x}^{(i)}$. At an untried design variable setting $\mathbf{x}$, the predicted objective function value is $\hat{y}$, which can be interpreted as a mean value at that location. Because we have not run a simulation at that location, there is a probability that the value is different. For both Kriging and RSM, a standard deviation $s$ can be calculated that reflects this uncertainty, see Santner et al. (2003) for Kriging and Myers and Montgomery (2002) for RSM. Figure 13 visualises this standard deviation at the untried design variable setting x. For Kriging, being an interpolative technique, the standard deviation equals 0 at the DOE points.

Validating the metamodels For each response, different metamodels have been fitted now. Metamodel validation techniques are employed to assess the accuracy of the different metamodels. Metamodel validation for RSM is based on analysis of variance (ANOVA) and residual plots, see e.g. Myers and Montgomery (2002). 


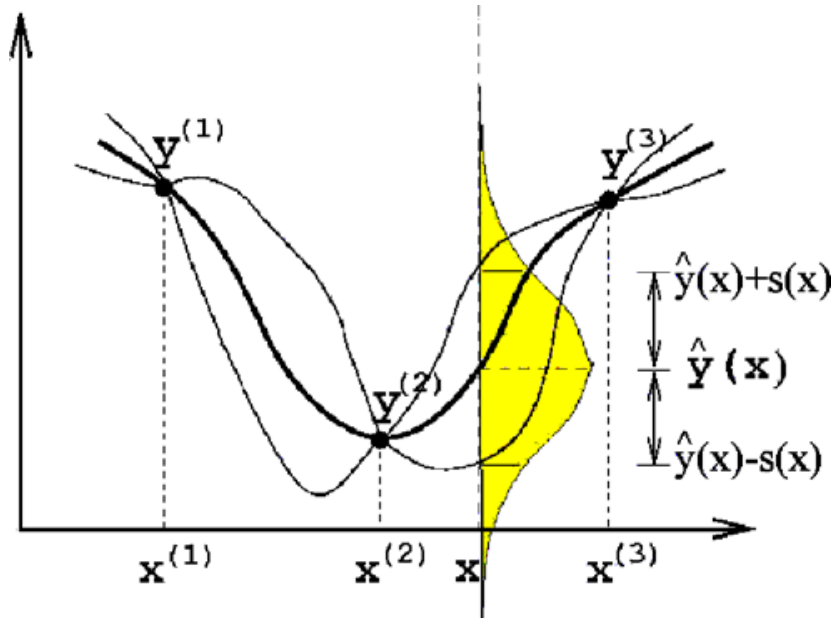

Fig. 13 A Kriging metamodel and its standard deviation

Metamodel validation for Kriging is based on cross validation (Bonte 2007; Bonte et al. 2007). Using these validation techniques, the best metamodels (either RSM or Kriging) for each response are selected and included as an approximation in the optimisation problem.

Optimisation The optimisation problem is subsequently optimised using a standard sequential quadratic programming (SQP) algorithm. In case constraints or Kriging metamodels are present in the final optimisation problem, there is a risk of ending up in a local optimum. This problem is overcome by initialising the SQP algorithm at multiple locations. This implies performing many function evaluations, but this is hardly a problem because both RSM and Kriging metamodels - being explicit mathematical functions - can be evaluated thousands of times within a second. The DOE points are used as initial locations for the SQP algorithm.

The obtained approximate optimum is finally checked by running one last FEM calculation with the approximated optimal settings of the design variables. Next to metamodel validation, the difference between the approximate objective function value and the real value of the objective function calculated by the last FEM run is an additional measure for the accuracy of the obtained optimum. If the user is not satisfied with the accuracy, the SAO algorithm allows for sequential improvement, as can be seen in Fig. 12.

Sequential improvement Three possible ways of sequential improvement have been presented and compared to each other by (Bonte et al. 2006; Bonte 2007). The basic idea of sequential improvement is to increase the accuracy of the optimum by adding new DOE points to the original spacefilling LHD. In this paper, we employ sequential improvement by minimising a merit function (SAO-MMF). The merit function is:

$f_{\text {merit }}=\hat{y}-w \cdot s$

where $\hat{y}$ and $s$ are, for both RSM and Kriging, given by metamodels from previous iterations of the algorithm, see Fig. 13. $w$ is a weight factor. If one selects $w=0$, the new DOE points equal the optima of the metamodel $\hat{y}$. If $w \rightarrow \infty$, the new DOE points are simply added in a spacefilling way. We found that $w=1$ provides a good compromise between both extreme cases. The merit function is minimised by the same multistart SQP algorithm introduced in the previous section. Again, the DOE points are used to initiate the SQP algorithm. Because the merit function in (1) is also a metamodel, minimising the merit function is very time-efficient.

Implementation The optimisation algorithm presented in Fig. 12 and the previous sections was implemented in MATLAB and can be used in combination with any FEM code for any metal forming process. It may also be applied to other applications for which performing many function evaluations is timeconsuming or otherwise prohibitive. For the fitting of the DACE/Kriging metamodels, use was made of the MATLAB Kriging toolbox implemented by Lophaven, Nielsen and Søndergaard (Nielsen 2002; Lophaven et al. 2002a, b). The efficiency of the algorithm has been assessed by comparing it to other optimisation algorithms and applying all algorithms to two forging processes, see (Bonte et al. 2006; Bonte 2007).

\section{Application to hydroforming}

We will demonstrate the proposed optimisation strategy - and the potential of optimisation in general by applying it to a simple hydroforming example, see Fig. 14.

\subsection{Modelling}

We follow the seven-step methodology for modelling the optimisation problem.

Step 1: Determine the appropriate optimisation situation Aim of optimisation is to design the manufacturing process to produce the part presented in Fig. 14. This is a process design type 1 situation.

Step 2: Select only the necessary responses The possible groups of responses for a process design type 1 situation are indicated in Fig. 10. These are DPs, defects 


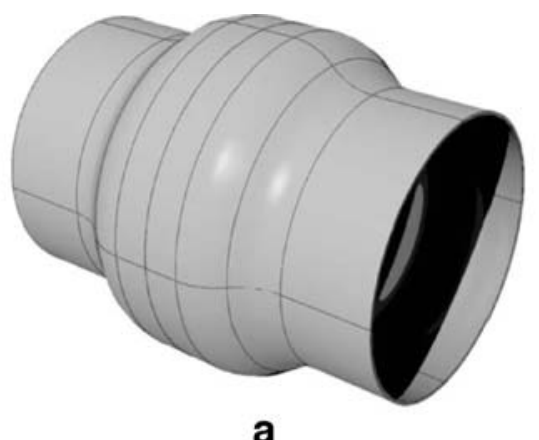

a

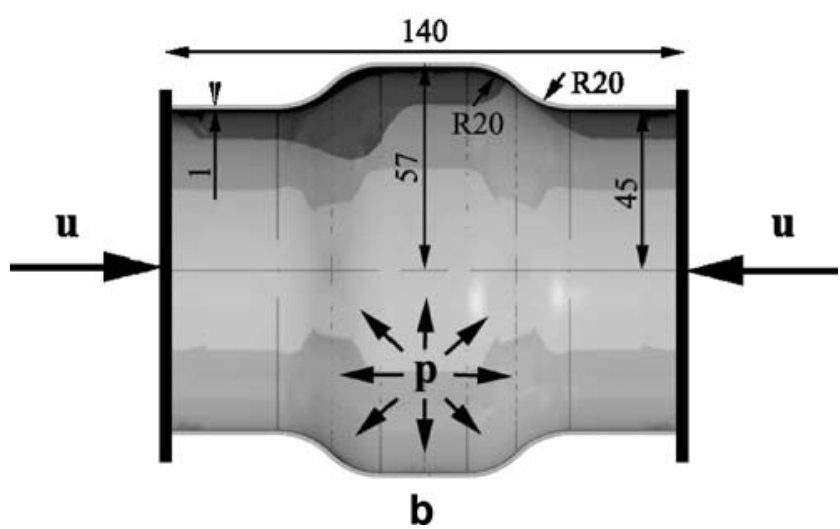

Fig. 14 a A simple hydroformed product; $\mathbf{b}$ dimensions

and costs. In this case we are interested in obtaining the intended part, which is contained in the category DPs. Defects and costs are, in this case, of less interest. Figure 7 assists in selecting the necessary responses. The part material is fixed; hence, the part geometry is the only relevant category. The essential geometrical parameters of the part are the wall thickness distribution and the outer shape accuracy of the part. Assuming the tools represent the perfect outer shape of the part, the latter response can be quantified as the distance between the part and the die after hydroforming.

Step 3: Select objective and implicit constraints The uniform wall thickness distribution is the objective, the constraint is the outer shape accuracy.

Step 4: Quantify the responses Table 1 is used to obtain the mathematical formulation of both responses. In this case, the objective function (thickness) is a noncritical nodal value. The constraint (distance between the final product and the die) is a critical nodal value. Following these observations, the final formulation is easily obtained from the table.

Step 5: Select possible design variables After having determined that we are dealing with a process design

type 1 situation, it is known (Fig. 10) that possible design variables are the process variables or PVs. The PVs have been subdivided further in the top-down structure in Fig. 8. What are we allowed to adapt? First of all, we are allowed to adapt the workpiece geometry. Figure 15a shows the initial tube and its geometrical parameters, which we can include as design variables. Because the part is formed by a one-stage hydroforming process, the tool geometry equals the final part geometry. In a process design type 1 situation, it is mostly not allowed to change the part geometry; hence, the tool geometry is also fixed. Following Fig. 8 to the material parameters, we can reason that the workpiece material is also fixed and that the tools are rigidly modelled in FEM. Hence, material-related design variables are not taken into account. Process parameters are the typical hydroforming load paths, which can be described by four design variables as can be seen in Fig. 15b. The most important design variable from the category "other process parameters" is the friction coefficient between part and tools, which can be influenced by adapting the lubrication.

The two geometrical parameters, four load path parameters and the friction coefficient add up to a sevendesign variable optimisation problem.

Step 6: Define the ranges on the design variables Upper and lower bounds have been defined on all seven design variables.

Step 7: Identify explicit constraints Explicit constraints describe impossible combinations of the design variables. The fact that the time when axial feeding ends should be larger than the time when it begins generates an explicit constraint. Another explicit constraint has been defined based on the convergence behaviour of the FEM calculations for certain combinations of the load path parameters.

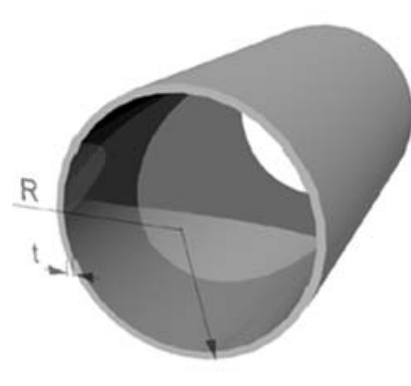

a

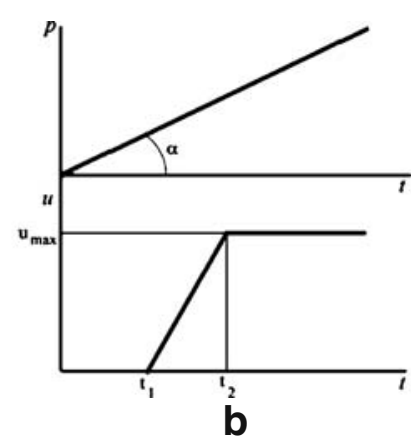

Fig. 15 a The workpiece and its geometrical parameters; b typical hydroforming load paths 


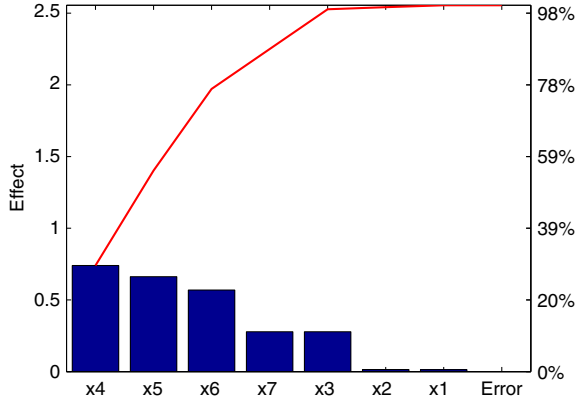

a

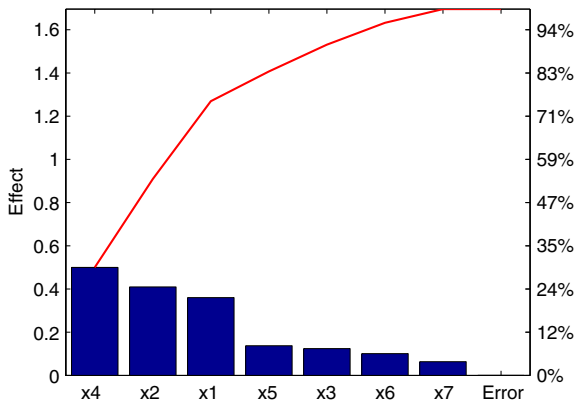

b

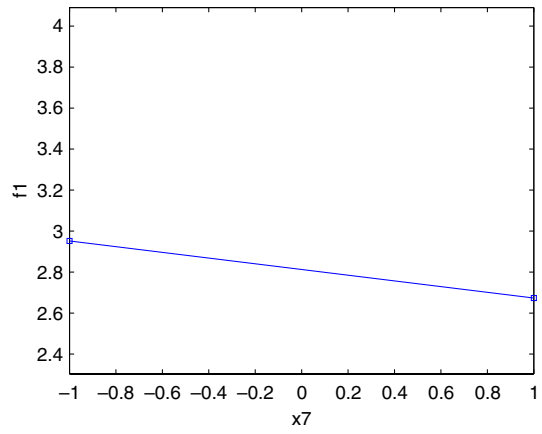

C

Fig. 16 a Pareto plot for the objective function; b Pareto plot for the implicit constraint; c effect plot

The structured seven-step methodology yielded the following mathematically formulated optimisation model:

$$
\begin{aligned}
\min f\left(t_{1}, t_{2}, \alpha, u_{\max }, R, t, \mu\right) & =\frac{\left\|h-h_{0}\right\|_{2}}{N} \\
\text { s.t. } \quad g_{\text {impl }} & =\max _{N}\left(d_{\text {tool-product }}\right) \leq 0 \\
g_{\text {expl1 }} & =t_{1}-t_{2} \leq 0 \\
g_{\text {expl2 }} & =u_{\max }-9\left(t_{1}-t_{2}\right) \leq 0 \\
0 \mathrm{~s} & \leq t_{1} \leq 5 \mathrm{~s} \\
2.5 \mathrm{~s} & \leq t_{2} \leq 10 \mathrm{~s} \\
9.5 \mathrm{MPa} / \mathrm{s} & \leq \alpha \leq 12 \mathrm{MPa} / \mathrm{s} \\
0 \mathrm{~mm} & \leq u_{\max } \leq 9 \mathrm{~mm} \\
40 \mathrm{~mm} & \leq R \leq 43.5 \mathrm{~mm} \\
0.8 \mathrm{~mm} & \leq t \leq 1.5 \mathrm{~mm} \\
0.10 & \leq \mu \leq 0.15
\end{aligned}
$$

where $h$ is the thickness throughout the final part, $h_{0}$ the final thickness defined by the part designer $(1 \mathrm{~mm}), N$ the number of nodes throughout the part and $d_{\text {tool-product }}$ the distance between final product and the die. $R$ and $t$ are the initial tube's radius and thickness, $t_{1}$ and $t_{2}$ are the time when axial feeding starts and stops, $u_{\max }$ is the amount of axial feeding and $\alpha$ is the increase in internal pressure. $\mu$ denotes the coefficient of friction.

\subsection{Screening}

Seven design variables is not that many; hence, it is possible to apply the SAO algorithm immediately. However, for demonstration purposes, we will perform screening anyway.

A $2_{\text {III }}^{(7-4)}$ fractional factorial DOE is applied, which implies that eight FEM simulations have been run to screen the importance of the seven design variables. A 2D axisymmetric FEM model has been made of the product. The in-house FE code DiekA has been used as FEM solver.

The resulting Pareto plot for the objective function is presented in Fig. 16a. Based on this plot, one may estimate that keeping the three most important variables $u_{\max }, R$ and $t$ and omitting the other four variables will still result in about $80 \%$ control over the objective function. A same Pareto plot has been generated for the implicit constraint, see Fig. 16b. $u_{\max }, t_{2}$ and $t_{1}$ were the most important variables for the filling of the die. It is important to keep control over all responses: if the most important variables are taken into account for the objective function only, it is possible that the less important variables are set to a level for which the implicit constraints are not satisfied. If the control over the constraints has been lost after screening, it is, during optimisation, not possible anymore to yield a feasible solution. Without losing too much control over

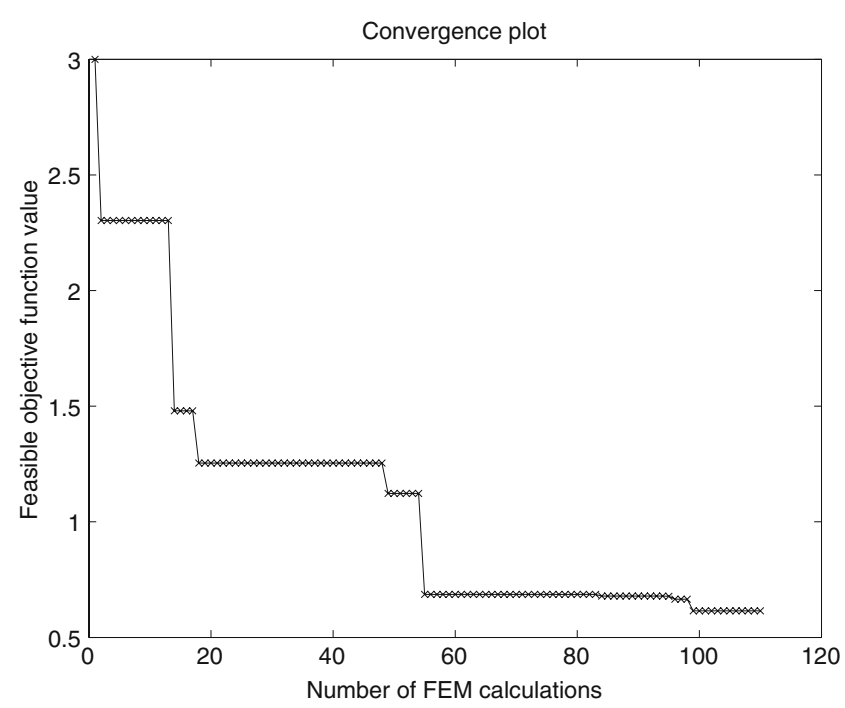

Fig. 17 Convergence of the optimisation algorithm 
Table 2 Optimised process settings

\begin{tabular}{lllllllll}
\hline$t_{1}$ & $t_{2}$ & $\alpha$ & $u_{\max }$ & $R$ & $t$ & $\mu$ & $f$ & $g_{1}$ \\
\hline 1.97 & 2.50 & 12 & 4.33 & 42.32 & 1.11 & 0.15 & 0.615 & -0.071 \\
\hline
\end{tabular}

both responses, it is, in this case, possible to reduce the number of design variables to five.

A question remaining is what to do with the two unimportant design variables $\mu$ and $\alpha$. For this, there are two options:

- Set them to a nominal value based on experience of the user.

- Use effect plots of the objective function.

In this case, we use the effect plots of the objective function. Figure 16c presents the effect of $\mu$ on the wall thickness. Because we are aiming to minimise the modelled objective function, one can best set $\mu$ to the maximal value. Analogously, one can set $\alpha$ to the maximal value, too.

\subsection{Solving}

The SAO algorithm has been applied to the reduced optimisation problem. Figure 17 shows the convergence behaviour of the algorithm. The simulations have been performed on 16 parallel processors; hence, the total calculation time was much shorter than running the 110 simulations sequentially. These 110 simulations include the eight screening simulations.

The optimised design variable settings are displayed in Table 2. The optimal objective function value is 0.615 and the negative value for the implicit constraint denotes that the final product properly fills out the die. Note the optimal initial tube thickness of $1.11 \mathrm{~mm}$. Con-

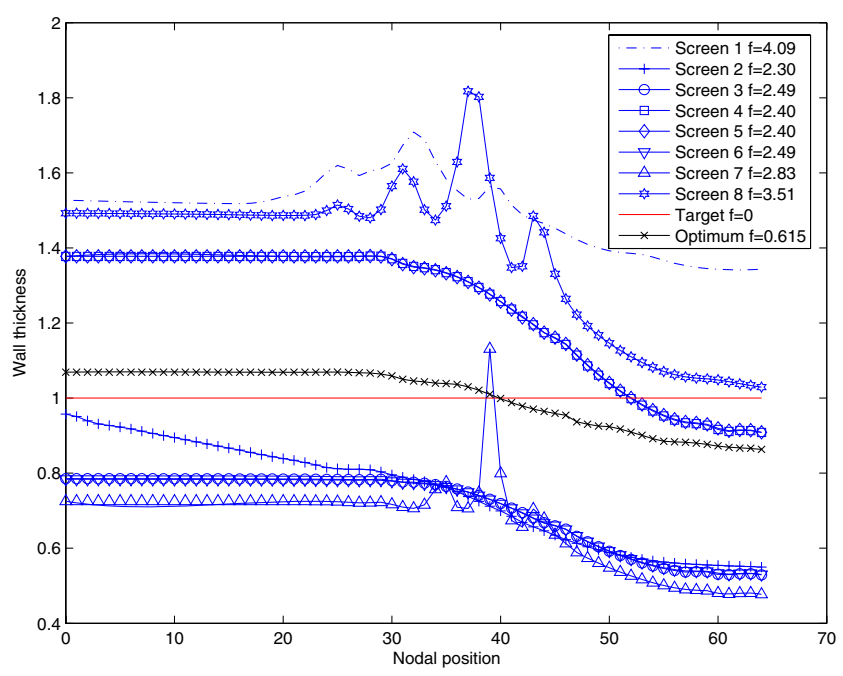

Fig. 18 Wall thickness distribution sidering the perfect wall thickness of $1 \mathrm{~mm}$ and material thinning due to inflation of the tube, this slightly thicker initial tube thickness is indeed the result one would intuitively expect to be optimal.

Figure 18 presents the wall thickness distribution throughout the final product for the perfect product (uniform wall thickness of $1 \mathrm{~mm}$ ), some arbitrary settings of the design variables (the eight screening calculations) and the optimised process. The optimised process yields a significantly better product, which demonstrates the high potential of optimisation in metal forming, and specifically the optimisation strategy proposed in Section 3.

\section{Conclusions}

A generally applicable optimisation strategy for metal forming processes has been proposed. It includes three stages: modelling, screening and solving.

The strategy includes a seven-step methodology for modelling optimisation problems in metal forming. In literature, this modelling is often done quite arbitrarily for a specific problem, product, or process. The proposed seven-step methodology is generally applicable to all kinds of optimisation problems, products and processes. Moreover, the structured methodology allows also non-optimisation specialists to generate a proper mathematical optimisation model of the metal forming problem they are facing.

The second stage is screening to reduce the optimisation problem size by selecting only the most important design variables. Resolution III fractional factorial design-of-experiment strategies provide a nice balance between effect estimation and efficiency. Analysis of variance and Pareto and effect plots are employed to estimate the importance of the design variables. Only the couple of most important design variables are taken into account in the final, reduced optimisation problem.

The reduced problem is subsequently solved by a generally applicable optimisation algorithm. This SAO algorithm has been developed by the authors to efficiently solve optimisation problems comprising timeconsuming function evaluations, e.g. FEM simulations for metal forming processes.

The optimisation strategy has been successfully applied to a hydroforming process. Modelling, screening and solving this metal forming problem demonstrated 
the potential of the optimisation of metal forming processes in general and, more specifically, the proposed optimisation strategy.

Acknowledgements This research has been carried out in the framework of the project "Optimisation of Forming Processes MC1.03162". This project is part of the research programme of the Netherlands Institute for Metals Research (NIMR). The industrial partners co-operating in this project are gratefully acknowledged for their useful contributions to this research.

Open Access This article is distributed under the terms of the Creative Commons Attribution Noncommercial License which permits any noncommercial use, distribution, and reproduction in any medium, provided the original author(s) and source are credited.

\section{References}

Abedrabbo N, Zafar N, Averill R, Pourboghrat F, Sidhu R (2004) Optimization of a tube hydroforming process. In: Proceedings of NUMIFORM, Columbus, OH, 13-17 June 2004

António C, Castro C, Sousa L (2004) Optimization of metal forming processes. Comput Struct 82:1425-1433

Aydemir A, de Vree J, Brekelmans W, Geers M, Sillekens W, Werkhoven R (2005) An adaptive simulation approach design for tube hydroforming processes. J Mater Process Technol 159:303-310

Ben Ayed L, Delamézière A, Batoz J, Knopf-Lenoir C (2004a) Optimization and control of the blankholder force in sheet metal stamping with application to deep drawing of a cylindrical cup. In: Proceedings of ECCOMAS, Jyväskylä, 24-28 July 2004

Ben Ayed L, Delamézière A, Batoz J, Knopf-Lenoir C (2004b) Optimization of the blankholder force with application to the numisheet' 02 deep drawing benchmark test b1. In: Proceedings of NUMIFORM, Columbus, OH, 13-17 June 2004

Ben Ayed L, Delamézière A, Batoz J, Knopf-Lenoir C (2005) Optimization of the blankholder force distribution in deep drawing. In: Proceedings of APOMAT, Morschach, 30-31 May 2005

Bonte MHA (2007) Optimisation strategies for metal forming processes. University of Twente, Enschede, The Netherlands, ISBN: 978-90-365-2523-7

Bonte M, van den Boogaard A, Huétink J (2005a) Metamodelling techniques for the optimisation of metal forming processes. In: Proceedings of ESAFORM, Cluj-Napoca, pp 155-158

Bonte M, van den Boogaard A, Huétink J (2005b) Solving optimisation problems in metal forming using finite element simulation and metamodelling techniques. In: Proceedings of APOMAT, Morschach, pp 242-251

Bonte M, Do T, Fourment L, van den Boogaard A, Huétink J, Habbal A (2006) A comparison of optimisation algorithms for metal forming processes. In: Proceedings of ESAFORM, Glasgow, pp 883-886

Bonte M, van den Boogaard A, Huétink J (2007) A metamodel based optimisation algorithm for metal forming processes. In: Banabic D. (ed) Advanced methods in material forming. Springer Verlag, Heidelberg, Germany, pp 55-72

Breitkopf P, Naceur H, Rassineux A, Villon P (2004) Optimizing metal forming processes using response surface approxima- tion and inverse approach surrogate model. In: Proceedings of ESAFORM, Trondheim, April 2004

Breitkopf P, Naceur H, Rassineux A, Villon P (2005) Moving least squares response surface approximation: Formulation and metal forming applications. Comput Struct 83(17-18):1411-1428

Byon S, Hwang S (2001) FEM-based process optimal design in steady-state metal forming considering strain-hardening. Comput Struct 79(14):1363-1375

Cao J, Li S, Xia Z, Tang S (2001) Analysis of an axisymmetric deep-drawn part forming using reduced forming steps. J Mater Process Technol 117(1-2):193-200

Carrino L, Giuliano G, Napolitano G (2003a) A posteriori optimisation of the forming pressure in superplastic forming processes by the finite element method. Finite Elem Anal Des 39:1083-1093

Carrino L, Giuliano G, Palmieri C (2003b) On the optimisation of superplastic forming processes by the finite-element method. J Mater Process Technol 143-144(1):373-377

Castro C, António C, Sousa L (2004) Optimisation of shape and process parameters in metal forging using genetic algorithms. Int J Mater Process Technol 146:356-364

Debray K, Sun Z, Radjai R, Guo Y, Dai L, Gu Y (2004) Optimum design of addendum surfaces in sheet metal forming process. In: Proceedings of NUMIFORM, Columbus, $\mathrm{OH}$, 13-17 June 2004

Di Lorenzo R, Filice L, Umbrello D, Micari F (2004) An integrated approach to the design of tube hydroforming processes: artificial intelligence, numerical analysis and experimental investigation. In: Proceedings of NUMIFORM, Columbus, OH, 13-17 June 2004

Do T, Fourment L, Laroussi M (2004) Sensitivity analysis and optimization algorithms for $3 \mathrm{D}$ forging process design. In: Proceedings of NUMIFORM, Columbus, OH, 13-17 June 2004

Duan X, Sheppard T (2002) Shape optimisation using FEA software: A V-shaped anvil as an example. J Mater Process Technol 120(1-3):426-431

Emmerich M, Giotis A, Özdemir M, Bäck T, Giannakoglou K (2002) Metamodel-assisted evolution strategies. In: Proceedings of the international conference on parallel problem solving from nature, Granada, 7-11 September 2002

Endelt B (2003) Least square optimization techniques applied on sheet metal forming. PhD thesis, Aalborg University, Aalborg

Endelt B, Nielsen K (2001) Least-square formulation of the object function, applied on hydro mechanical tube forming. In: Proceedings of SheMet, Leuven, 2-4 April 2001

Fann K, Hsiao P (2003) Optimization of loading conditions for tube hydroforming. J Mater Process Technol 140(1-3): $520-524$

Fourment L, Do T, Habbal A, Bouzaiane M (2005a) Gradient, non-gradient and hybrid algorithms for optimizing 2D and 3D forging sequences. In: Proceedings of ESAFORM, Cluj-Napoca, April 2005

Fourment L, Do T, Habbal A, Bouzaiane M (2005b) Optimization of forging sequences using gradient, non-gradient and hybrid algorithms. In: Proceedings of APOMAT, Morschach, 30-31 May 2005

Jansson T (2002) Optimization of sheet metal forming processes. Licentiate thesis, Universitet Linköping, Linköping

Jansson T, Andersson A, Nilsson L (2005) Optimization of drawin for an automotive sheet metal part-an evaluation using surrogate models and response surfaces. J Mater Process Technol 159:426-234 
Jirathearanat S, Altan T (2004) Optimization of loading paths for tube hydroforming. In: Proceedings of NUMIFORM, Columbus, OH, 13-17 June 2004

Johnson K, Nguyen B, Dawies R, Grant G, Khaleel M (2004) A numerical process control method for circular-tube hydroforming prediction. Int J Plast 20:1111-1137

Kim N, Choi K, Chen J (2001a) Die shape design optimization of sheet metal stamping process using meshfree method. Int J Numer Methods Eng 51:1385-1405

Kim Y, Lee J, Hong S (2001b) Optimal design of superplastic forming processes. J Mater Process Technol 112(2-3): $166-173$

Kleinermann J (2001) Identification paramétrique et optimisation des procédés de mise à forme par problèmes inverses. $\mathrm{PhD}$ thesis, Université de Liège, Liège (in French)

Kleinermann J, Ponthot J (2003) Parameter identification and shape/process optimization in metal forming simulation. J Mater Process Technol 139(1-3):521-526

Koc M, Allen T, Jiratheranat S, Altan T (2000) Use of FEA and design of experiments to establish design guidelines for simple hydroformed parts. Int $J$ Mach Tools Manuf 40(15):2249-2266

Labergère C, Gelin J (2004a) Comparison between optimization and control strategies to determine command laws in hydroforming processes. In: Proceedings of ESAFORM, Trondheim, April 2004

Labergère C, Gelin J (2004b) New strategies for optimal control of command laws for tube hydroforming processes. In: Proceedings of NUMIFORM, Columbus, OH, 13-17 June 2004

Labergère C, Lejeune A, Gelin J (2004) Control of damage in flanges hydroforming. In: Proceedings of ECCOMAS, Jyväskylä, 24-28 July 2004

Lenoir H, Boudeau N (2003) An optimization procedure for springback compensation. In: Proceedings of ESAFORM, Salerno, 28-30 April 2003

Liew K, Tan H, Ray T, Tan M (2004) Optimal process design of sheet metal forming for minimum springback via an integrated neural network evolutionary algorithm. Struct Multidisc Optim 26:284-294

Lin Z, Juchen X, Xinyun W, Guoan H (2002) Sensitivity based optimization of sheet metal forming tools. J Mater Process Technol 124(3):319-328

Lin Z, Juchen X, Xinyun W, Guoan H (2003) Optimization of die profile for improving die life in the hot extrusion process. J Mater Process Technol 142(3):659-664

Lophaven S, Nielsen H, Søndergaard J (2002a) Aspects of the MATLAB toolbox DACE. Technical report IMM-REP2002-13. Technical University of Denmark-Department of Informatics and Mathematical Modelling, Lyngby

Lophaven S, Nielsen H, Søndergaard J (2002b) DACE - A MATLAB Kriging Toolbox. Technical report IMM-TR2002-12. Technical University of Denmark-Department of Informatics and Mathematical Modelling, Lyngby

McKay M, Beckman R, Conover W (1979) A comparison of three methods for selecting values of input variables in the analysis of output from a computer code. Technometrics 21:239-245

Myers R, Montgomery D (2002) Response surface methodology: process and product optimization using designed experiments, 2nd edn. Wiley, New York

Naceur H, Guo Y, Batoz J, Knopf-Lenoir C (2001) Optimization of drawbead restraining forces and drawbead design in sheet metal forming process. Int J Mech Sci 43(10):2407-2434
Naceur H, Breitkopf P, Knopf-Lenoir C, Rassineux A, Villon P (2003) Response surface methods for the optimal design of the initial blank in sheet metal forming process. In: Proceedings of ESAFORM, Salerno, 28-30 April 2003

Naceur H, Ben-Elechi S, Batoz J (2004a) The inverse approach for the design of sheet metal forming parameters to control springback effects. In: Proceedings of ECCOMAS, Jyväskylä, 24-28 July 2004

Naceur H, Ben-Elechi S, Knopf-Lenoir C, Batoz J (2004b) Response surface methodology for the design of sheet metal forming parameters to control springback effects using the inverse approach. In: Proceedings of NUMIFORM, Columbus, OH, 13-17 June 2004

Naceur H, Delamézière A, Batoz J, Guo Y, Knopf-Lenoir C (2004c) Optimization of drawbead restraining forces and drawbead design in sheet metal forming process. J Mater Process Technol 146:250-262

Nielsen H (2002) DACE, a MATLAB Kriging toolbox. http://www.imm.dtu.dk/ hbn/dace/

Ohata T, Nakamura Y, Katayama T, Nakamachi E (2003) Development of optimum process design system for sheet fabrication using response surface method. J Mater Process Technol 143-144(1):667-672

Palaniswamy H, Ngaile G, Altan T (2004) Optimization of blank dimensions to reduce springback in the flexforming process. J Mater Process Technol 146:28-34

Papalambros P, Wilde D (2000) Principles of optimal design. Cambridge University Press, New York

Ponthot J, Kleinermann J (2005) Optimisation methods for initial/tool shape optimisation in metal forming processes. Int $\mathrm{J}$ Veh Des 39(1-2):14-24

Poursina M, António C, Parvizian J, Sousa L, Castro C (2003) Eliminating folding defect in forging parts using a genetic algorithm. In: Proceedings of ESAFORM, Salerno, 28-30 April 2003

Ray P, Mac Donald B (2004) Determination of the optimal load path for tube hydroforming processes using a fuzzy load control algorithm and finite element analysis. Finite Elem Anal Des 41:173-192

Repalle J, Grandhi R (2004) Optimum design of forging process parameters and preform shape under uncertainties. In: Proceedings of NUMIFORM, Columbus, OH, 13-17 June 2004

Revuelta A, Larkiola J (2004) Applying neural networks in the final thickness optimisation of a thin hydroformed part. In: Proceedings of ECCOMAS, Jyväskylä, 24-28 July 2004

Sacks J, Schiller S, Welch W (1989a) Design for computer experiments. Technometrics 31:41-47

Sacks J, Welch W, Mitchell T, Wynn H (1989b) Design and analysis of computer experiments. Stat Sci 4:409-423

Sahai A, Schramm U, Buranathiti T, Chen W, Cao J, Xia C (2004) Sequential optimization and reliability assessment method for metal forming processes. In: Proceedings of NUMIFORM, Columbus, OH, 13-17 June 2004

Santner T, Williams B, Notz W (2003) The design and analysis of computer experiments. Springer, Berlin Heidelberg New York

Schenk O, Hillmann M (2004) Optimal design of metal forming die surfaces with evolution strategies. Comput Struct 82:1695-1705

Sheng Z, Jirathearanat S, Altan T (2004) Adaptive FEM simulation for prediction of variable blank holder force in conical cup drawing. Int J Mach Tools Manuf 44:487-494

Shi X, Chen J, Peng Y, Ruan X (2004) A new approach of die shape optimization for sheet metal forming processes. J Mater Process Technol 152:35-42 
Sillekens W, Werkhoven R (2001) Hydroforming processes for tubular parts: optimisation by means of adaptive and iterative FEM simulation. Int J Form Process 4(3-4):377-393

Sousa L, António C, Castro C (2003) Optimisation of multistep hot forging operations. In: Proceedings of ESAFORM, Salerno, 28-30 April 2003

Strano M, Carrino L (2004) Adaptive selection of loads during FEM simulation of sheet forming processes. In: Proceedings of NUMIFORM, Columbus, OH, 13-17 June 2004

Thiyagarajan N, Grandhi R (2004) 3-D preform shape optimization in metal forming. In: Proceedings of NUMIFORM, Columbus, OH, 13-17 June 2004
Weyler R, Neamtu L, Duffett G (2004) An approach to the computer simulation of metal forming processes. In: Proceedings of ECCOMAS, Jyväskylä, 24-28 July 2004

Yang J, Jeon B, Oh S (2001) Design sensitivity analysis and optimization of the hydroforming process. J Mater Process Technol 113:666-672

Yang K, El-Haik B (2003) Design for six sigma; a roadmap for product development. McGraw-Hill, New York

Zhao G, Ma X, Zhao X, Grandhi R (2004) Studies on optimization of metal forming processes using sensitivity analysis methods. J Mater Process Technol 147: $217-228$ 